\title{
Pharmacokinetics of antimicrobial agents in anuric patients during continuous venovenous haemofiltration
}

\author{
B. Joos, M. Schmidli and G. Keusch \\ Department of Medicine, University Hospital, Zürich, Switzerland
}

\begin{abstract}
Background. The optimal drug dosing in anuric patients undergoing continuous haemofiltration is a difficult task. More pharmacokinetic data is needed to derive practical guidelines for dosage adjustments.

Methods. Drug elimination of various antimicrobial agents (amikacin, amoxycillin, ceftazidime, ciprofloxacin, flucloxacillin, imipenem, netilmicin, penicillin $\mathrm{G}$, piperacillin, sulphamethoxazole, tobramycin, vancomycin) was studied in 24 patients with acute renal failure treated by pump-assisted continuous venovenous haemofiltration (CVVH). Concentrations of serial blood and ultrafiltrate samples were determined by HPLC or by fluorescence polarization immunoassay. Total body clearance (CL) and haemofilter clearance $\left(\mathrm{CL}_{\mathrm{f}}\right)$ rates were determined by standard modelindependent equations. Data from published literature on fractions not bound to proteins $\left(f_{u}\right)$, non-renal drug clearance fractions $\left(Q_{o}\right)$, and normal clearance values $\left(\mathrm{CL}_{\mathrm{n}}\right)$ were used to derive a pharmacokinetic model, taking into account drug removal by ultrafiltration and by non-renal clearance.

Results. A total of 37 treatment periods was studied. Blood flow through the haemofilters was $100 \mathrm{ml} / \mathrm{min}$ resulting in an average ultrafiltrate flow rate (UFR) of $13.2 \pm 4.6$ (range 3.2-22.1) $\mathrm{ml} / \mathrm{min}$. Acceptable correlations of calculated and measured haemofilter clearances and total body clearances were obtained.

Conclusions. Total body clearance in anuric patients during CVVH is predictable from drug properties, which are generally known. The individual dosage requirements may be calculated by multiplying $\mathrm{Q}_{\mathrm{o}}+\mathrm{f}_{\mathrm{u}} \cdot \mathrm{UFR} / \mathrm{CL}_{\mathrm{n}}$ with the dose considered appropriate in the absence of renal impairment.
\end{abstract}

Key words: acute renal failure; antimicrobial agents; continuous venovenous haemofiltration; drug dosage; pharmacokinetics

Correspondence and offprint requests to: Dr Beda Joos, Division of Infectious Diseases. Department of Medicine, University Hospital Zürich, CH-8091 Zürich, Switzerland.

\section{Introduction}

Continuous haemofiltration is a well-established tool in the treatment of patients with renal failure. However, despite good control of uraemia, acute renal failure is often associated with a high mortality rate [1]. Septicaemia frequently complicates the disease progression, and it has been identified as a major predictor of a poor outcome. Optimal drug dosing is thus essential in this situation but pharmacokinetic data during continuous renal replacement therapy are scarce. Complex mathematical models have been proposed [2,3], but their application in clinical practice is limited. The current methods to estimate drug removal through the haemofilter rely on sieving coefficients, i.e. average filtrate to blood concentration ratios [2-5]. These values, however, must be determined previously for each drug.

The aim of this study is to investigate the pharmacokinetics of various antimicrobial agents in patients with acute renal failure undergoing pump-assisted continuous venovenous haemofiltration (CVVH) and to derive practical guidelines for dosage adjustments.

\section{Subjects and methods}

\section{Selection of patients}

Anuric patients undergoing continuous renal replacement therapy and receiving antimicrobial chemotherapy were considered eligible for inclusion in the study. Drug clearance rates were measured during the CVVH in a total of 24 patients with acute renal failure due to various causes.

\section{Haemofiltration}

Pump-assisted CVVH was performed by a double-lumen subclavian catheter [1]. Blood flow through the polyamide hollow-fibre membrane filters (Gambro FH66) was set at $100 \mathrm{ml} / \mathrm{min}$. The replacement fluid (Ringer's lactate) was administered in pre-dilution or post-dilution mode. The ultrafiltrate flow rate (UFR) was continuously monitored throughout treatment. 
Table 1. Dosage and median pharmacokinetic parameters observed in 24 patients during contınuous venovenous haemofiltration

\begin{tabular}{|c|c|c|c|c|c|c|c|}
\hline Drug & $\begin{array}{l}\text { Number of } \\
\text { patients }\end{array}$ & $\begin{array}{l}\text { Number of } \\
\text { intervals }\end{array}$ & $\begin{array}{l}\text { Dose } \\
(\mathrm{mg})\end{array}$ & $\begin{array}{l}\text { Interval } \\
\text { (h) }\end{array}$ & $\begin{array}{l}\text { Half-life } \\
\text { (h) }\end{array}$ & $\begin{array}{l}\text { CL-total } \\
(\mathrm{ml} / \mathrm{min})\end{array}$ & $\begin{array}{l}\text { CL-filter } \\
(\mathrm{ml} / \mathrm{min})\end{array}$ \\
\hline Amikacin & 2 & 2 & $600-800$ & 24 & 15.6 & 26 & 16 \\
\hline Amoxycillin & 3 & 3 & $600-1200$ & $6-12$ & 5.8 & 38 & 10 \\
\hline Ceftazidime & 6 & 13 & $500-1000$ & $6-13$ & 13.2 & 20 & 5 \\
\hline Ciprofloxacin & 2 & 3 & 200 & $6-12$ & 7.5 & 231 & 14 \\
\hline Flucloxacillin & 3 & 6 & $1000-2000$ & $4-39$ & 9.9 & 27 & 2 \\
\hline Imipenem & 1 & 2 & 500 & 12 & 3.0 & 139 & 4 \\
\hline Netilmicin & 6 & 10 & $40-200$ & $24-72$ & 13.9 & 20 & 12 \\
\hline Penicillin-G & 1 & 2 & 1198 & 4 & 12.6 & 51 & 11 \\
\hline Piperacillin & 8 & $1 \overline{6}$ & $1000-4000$ & $4-12$ & 10.6 & 56 & 10 \\
\hline Sulphamethoxazole & $1^{a}$ & 1 & 400 & 12 & 9.9 & 65 & 10 \\
\hline Tobramycin & 1 & 2 & 40 & 24 & 9.2 & 13 & 9 \\
\hline Vancomycin & 3 & 4 & $500-1000$ & $24-43$ & 23.1 & 34 & 17 \\
\hline Total, range & $37^{b}$ & 64 & $40-4000$ & $4-72$ & $2.3-139$ & $9-286$ & $1-27$ \\
\hline
\end{tabular}

${ }^{a}$ Trimethoprim concentrations in haemofiltrate were below the limit of detection $(<0.2 \mathrm{mg} / 1)$ in this patient.

b Therapy consisted of $1-3$ different drugs in individual patients, resulting in a total of 37 treatment periods.

\section{Dosage and administration}

Antibiotics were administered intravenously either alone or in combination (Table 1). The choice of antimicrobial therapy for each patient was based on clinical judgement only. The dosing interval was chosen according to the presumed halflife of the respective drug.

Up to three different drugs were administered to each patient during the study, resulting in a total of 37 evaluable treatment periods. In many cases, several subsequent dosing intervals per drug and patient were studied ( 64 totally) in order to obtain more reliable data and to ensure that a steady state was reached.

\section{Specimen collection}

Serial blood samples and pooled ultrafiltrate were obtained at various time points during the steady state. On the average, $11 \pm 7$ specimens were collected during each of the 37 treatment periods. The optimal timing of sample collection was determined individually depending on the drug to be studied and on the actual length of the dosing interval. Concentrations of imipenem were determined immediately due to its limited stability, while aminoglycoside and vancomycin levels were assayed within $24 \mathrm{~h}$. All other serum and haemofiltrate samples were stored together with appropriate control standards at $-70^{\circ} \mathrm{C}$ for up to 3 months before being measured in batch.

\section{Assay methods}

Concentrations of beta-lactam antibiotics, ciprofloxacin, and sulphamethoxazole were determined by HPLC whereas the aminoglycosides and vancomycin were measured by a fluorescence polarization immunoassay (Abbott TDX).

\section{Pharmacokinetic analysis}

The timing of the doses, dosing intervals, duration of infusion, and the volume of the ultrafiltrate produced per time unit were recorded for each patient. A serum concentration versus time profile was plotted for each dosing interval and the area under the curve (AUC) was calculated by the log- trapezoidal method. The amount of drug excreted through the haemofilter during one dosing interval was calculated from the volume of collected ultrafiltrate and the drug concentration in the ultrafiltrate. Standard model-independent equations were applied to calculate total body clearance $(\mathrm{CL}=$ Dose/AUC) and filter clearance (excreted amount/AUC).

Selected drug properties such as protein binding (unbound fraction $f_{u}$ ), non-renal drug clearance fraction $\left(Q_{o}\right)$, and normal clearance values $\left(\mathrm{CL}_{\mathrm{n}}\right)$ were derived from published literature [6-8] (Table 2).

\section{Statistical methods}

The correlations of calculated and measured clearances were determined by standardized principal component analysis [9], and by distribution independent regression analysis according to Passing and Bablok [10,11]. The latter model estimates confidence intervals for slope and intercept and tests for linearity by cusum statistics and by the run test. The performance of the prediction method was determined

Table 2. Selected drug properties used to predict total body clearance and haemofilter clearance [6-8]

\begin{tabular}{llll}
\hline Drug & $\mathrm{f}_{\mathrm{u}}{ }^{\mathrm{a}}$ & $\mathrm{Q}_{\mathrm{o}}{ }^{\mathrm{a}}$ & $\begin{array}{l}\mathrm{CL}_{\mathrm{n}}{ }^{\mathrm{a}} \\
(\mathrm{ml} / \mathrm{min})\end{array}$ \\
\hline Amikacin & 0.96 & 0.02 & 92 \\
Amoxycillin & 0.87 & 0.06 & 372 \\
Ceftazidime & 0.85 & 0.05 & 132 \\
Ciprofloxacin & 0.7 & 0.5 & 500 \\
Flucloxacillin & 0.2 & 0.3 & 83 \\
Imipenem & 0.75 & 0.3 & 233 \\
Netilmicin & 0.96 & 0.01 & 92 \\
Penicillin-G & 0.6 & 0.08 & 500 \\
Piperacillin & 0.84 & 0.25 & 182 \\
Sulphamethoxazole & 0.6 & 0.8 & 26 \\
Tobramycin & 1.0 & 0.02 & 77 \\
Vancomycin & 0.9 & 0.05 & 77 \\
Range & $0.2-1.0$ & $0.01-0.8$ & $26-500$ \\
\hline
\end{tabular}

${ }^{\mathrm{a}} \mathrm{f}_{\mathrm{u}}$, unbound fraction; $\mathrm{Q}_{0}$, non-renal drug clearance fraction; $\mathrm{CL}_{\mathrm{n}}$, normal clearance. 
by calculating bias and precision (root of mean squared differences) according to Sheiner and Beal [12].

\section{Results}

Twenty-four patients were included in the study. Approximately $50 \%$ of the patients experienced an acute renal failure due to various internal medical causes, e.g. septic shock, pneumonia, ARDS, pancreatitis, hepatorenal syndrome. One-third of the patients suffered from postoperative acute renal failure following either renal transplantation, combined renal and pancreas transplantation, or cardiac surgery. The remaining patients had multiple traumatic lesions or traumatic brain injury. Half of the patients were on the internal and half on the surgical intensive care unit. All patients were attended by a nephrologist.

The antimicrobial therapy used consisted of 12 different antimicrobial agents given in a wide variety of dosages either alone or in combination (Table 1). Serum and filtrate kinetics were studied during a total of 37 treatments ( 64 dosing intervals). The average ultrafiltrate flow rate was $13.2 \pm 4.6 \mathrm{ml} / \mathrm{min}$ (mean $\pm \mathrm{SD}$, range 3.2-22.1).

Using the fundamental pharmacokinetic drug parameters observed in individuals without renal failure (Table 2), a theoretical model was derived which could be used to predict the clearance values. The measured haemofilter clearances and total body clearances were in good agreement with $f_{u} \cdot U F R$ and with $\mathrm{f}_{\mathrm{u}} \cdot \mathrm{UFR}+\mathrm{Q}_{\mathrm{o}} \cdot \mathrm{Cl}_{\mathrm{n}}$ respectively. Figures 1 and 2 illustrate the distribution of the observed values. The quantity $f_{u} \cdot U F R$ was a good predictor of filter clearance (Table 3). The majority of the measurements deviated by less than $15 \%$. Total body clearance was underestimated by $\mathrm{f}_{\mathrm{u}} \cdot \mathrm{UFR}+\mathrm{Q}_{0} \cdot \mathrm{Cl}_{\mathrm{n}}$ by an average of $7.6 \mathrm{ml} / \mathrm{min}$. Deviations were $<33 \%$, however, in the majority of the 37 treatment periods.

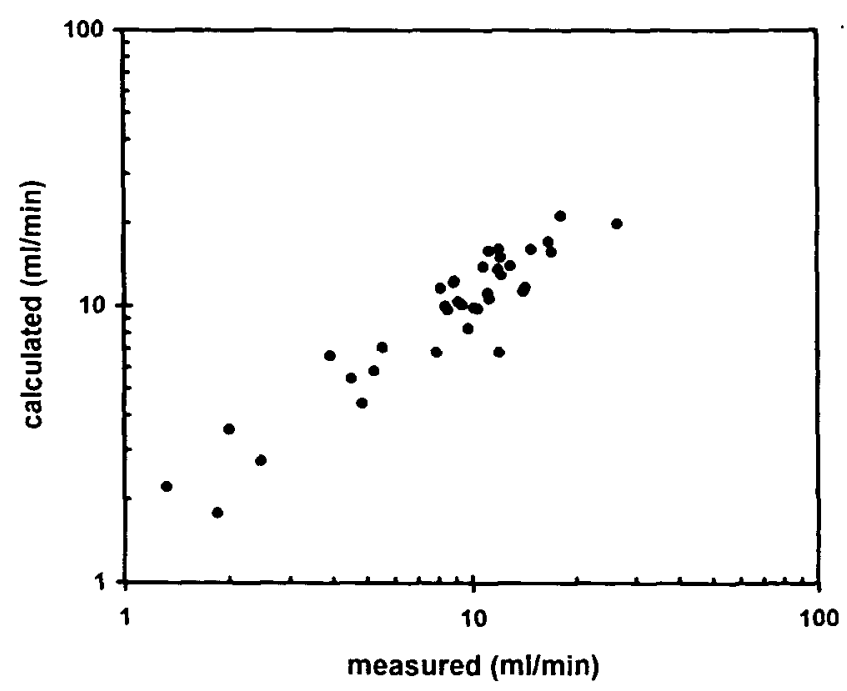

Fig. 1. Measured and calculated haemofilter clearance rates of 12 different drugs during continuous venovenous haemofiltration (calculated values were obtained from $f_{n} \cdot$ UFR).

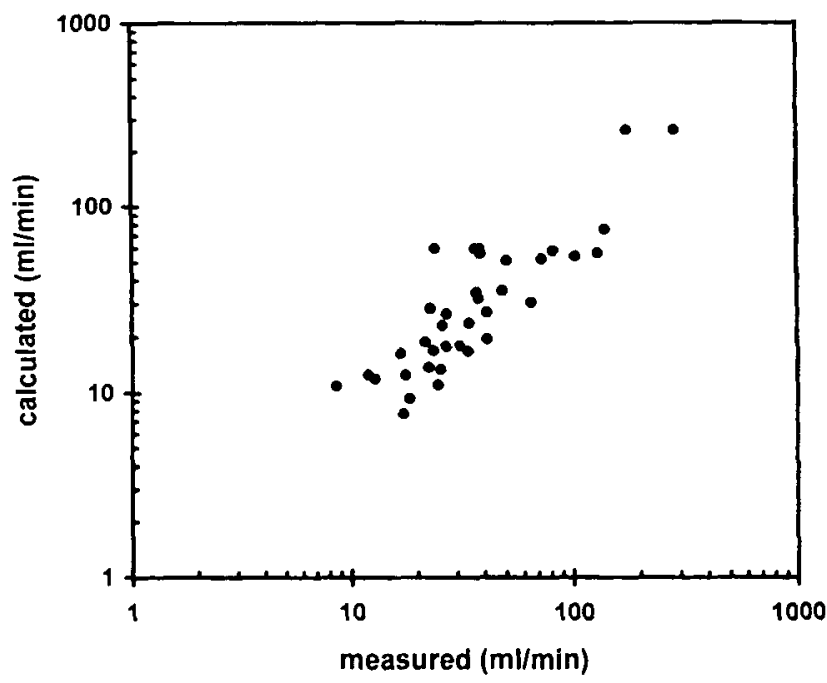

Fig. 2. Measured and calculated total body clearance rates of 12 different drugs during continuous venovenous haemofiltration (calculated values were obtained from $f_{u} \cdot U F R+Q_{0} \cdot C L_{n}$ ).

Table 3. Comparison of measured and calculated haemofilter clearance and total body clearance values during 37 treatment periods

\begin{tabular}{|c|c|c|}
\hline Method & Filter clearance ${ }^{a}$ & Total clearance ${ }^{a}$ \\
\hline \multicolumn{3}{|c|}{ Predictive performance [12]: } \\
\hline $\begin{array}{l}\text { bias }(\mathrm{ml} / \mathrm{min}) \\
\text { precision }\left(\mathrm{rmse}^{\mathrm{b}}\right)\end{array}$ & $\begin{array}{l}0.6(-0.2,1.4) \\
2.4(1.7,3.0)\end{array}$ & $\begin{array}{c}-7.6(-16.4,1.1) \\
27.0(14.0,35.6)\end{array}$ \\
\hline \multicolumn{3}{|c|}{ Standardized principal component analysis [9]: } \\
\hline $\begin{array}{l}\text { slope } \\
\mathrm{y} \text {-intercept }(\mathrm{ml} / \mathrm{min})\end{array}$ & $\begin{array}{l}0.94 \\
1.3\end{array}$ & $\begin{aligned} & 1.03 \\
- & 8.9\end{aligned}$ \\
\hline \multicolumn{3}{|c|}{ Distribution-independent regression analysis $[10,11]$ : } \\
\hline $\begin{array}{l}\text { slope }^{c} \\
y \text {-intercept } \\
\end{array}$ & $\begin{array}{l}1.04(0.87,1.24) \\
0.56(-1.3,2.0)\end{array}$ & $\begin{aligned} & 0.81(0.57,1.05) \\
- & 2.0(-9.9,5.7)\end{aligned}$ \\
\hline
\end{tabular}

a $95 \%$ confidence intervals are indicated in parentheses.

b Root of mean squared prediction error.

- No statistically significant differences of slopes from 1 or of intercepts from $0(P<0.05)$ and no significant deviation from linearity as determined by the cusum and run tests $(P>0.1)$.

Comparison of the measured and calculated values for both haemofilter and total body clearance by distribution-independent linear regression analysis yielded slopes of approximately 1 and $y$-intercepts near $0 \mathrm{ml} / \mathrm{min}$ (Table 3). No significant deviation from linearity was found by the cusum and run tests $(P>0.1)$.

\section{Discussion}

The existing mathematical models used to estimate drug elimination during continuous renal replacement therapy have recently been reviewed by ReetzeBonorden et al. [5]. Sieving coefficients are frequently used to characterize the ability of a drug to pass through the haemofilter membrane. These values, how- 
ever, have not been measured for all drugs used in patients with acute renal failure.

The approach presented in this study has the advantage that all parameters needed to estimate drug clearance are readily available. The convective transport from blood to ultrafiltrate was calculated from ultrafiltrate flow rate and unbound fraction alone, and the non-renal clearance was assumed to be the same as in patients without renal impairment. This simple model is far from being perfect. It is evident that protein binding may be altered in anuric patients and nonrenal elimination may be impaired in these severely ill patients. Moreover, possible interactions due to multiple drug therapy may be responsible for pharmacokinetic alterations. It is conceivable also that a reduced hepatic clearance may be in part compensated by lower protein binding and consequently increased haemofilter clearance and vice versa.

Various statistical methods were used to compare predicted with measured haemofilter and total body clearances. Evaluation of predictive performance [12] has become usual practice; however, this method demands an independent variable presumed to be free of experimental error. Standardized principal component analysis [9] is a multivariate procedure, but normal distribution of the values is required. Regression analysis, according to Passing and Bablok [10,11], may overcome these drawbacks since it does not depend on an error-free variable or on rigid distributional requirements. In addition, this procedure tests for a linear relationship between the two variables, confidence limits are given for the slope and the intercept, and the influence of outliers is minimized.

The 12 drugs studied exhibited marked differences in their protein binding as well as in their non-renal contribution to total clearance. In addition wide ranges of dose, dosing intervals, and ultrafiltration rates were covered. Nevertheless in most patients the proposed formula $\mathrm{f}_{\mathrm{u}} \cdot \mathrm{UFR}+\mathrm{Q}_{\mathrm{o}} \cdot \mathrm{CL}_{\mathrm{n}}$ provided a reliable estimate of the total drug clearance. The individual dosage requirements may be calculated in proportion to the ratio of total body clearance observed during CVVH and the normal clearance rate found in patients without renal impairment. Consequently the following formula may be used to predict the maintenance dose during renal replacement therapy.

Dose $=D_{n} \cdot\left(Q_{0}+f_{u} \cdot U F R / C L_{n}\right)$

where $\mathrm{D}_{n}$ denotes normal dose, $\mathrm{CL}_{\mathrm{n}}$ normal clearance, $Q_{0}$ non-renal clearance fraction, $f_{u}$ unbound fraction, and UFR ultrafiltrate flow rate.

In conclusion, the results of the present study allow practical dosage recommendations to be made for anuric patients undergoing CVVH. Most drugs used in clinical practice have relatively low molecular weights, so that similar mechanisms of clearance during CVVH would be expected. It is therefore anticipated that the same dosage calculations will also be appropriate for the majority of other therapeutic agents. Monitoring serum concentrations, however, remains mandatory when using drugs which possess a narrow therapeutic range. Prospective studies are needed to evaluate the efficiency of these dosage guidelines and to study specifically whether the desired drug concentrations are achieved.

Acknowledgements. The authors thank the nursing staff of the medical intensive care unit of the University Hospital Zürich for their invaluable assistance, and Peter Gowland for his helpful review of this manuscript.

\section{References}

1. Keusch G, Schreier P, Binswanger U. Outcome in critucally ill patients with acute renal failure treated by continuous hemofiltration. Contrib Nephrol 1991; 93: 57-60

2. Vincent $\mathrm{HH}$, van Ittersum $\mathrm{FJ}$, Akcahuseyin $\mathrm{E}$, Vos $\mathrm{MC}$, van Duyl WA, Schalekamp MA. Solute transport in continuous arteriovenous hemodiafiltration: a new mathematical model applied to clinical data. Blood Purif 1990; 8: 149-159

3. Kroh UF, Dehne M, El Abed K, Feussner KD, Hofmann W, Lennartz $\mathrm{H}$. Drug dosage during continuous hemofiltration: pharmacokinetics and practical implications. Contrib Nephrol 1991; 93: $127-130$

4. Golper TA, Wedel SK, Kaplan AA, Saad AM, Donta ST, Paganini EP. Drug removal during continuous arteriovenous hemofiltration: theory and clinical observations. Int J Artif Organs 1985; 8: 307-312

5. Reetze-Bonorden P, Böhler J, Keller E. Drug dosage in patients during continuous renal replacement therapy. Pharmacokinetic and therapeutic considerations. Clin Pharmacokinet 1993; 24: 362-379

6. Vozeh S, Schmidlin O, Taeschner W. Pharmacokinetic drug data. Clin Pharmacokinet 1988; 15: 254-282

7. Dettli L. Pharmakokinetische Daten für die Dosisanpassung. In: Arzneimittel - Kompendium der Schweiz, Registerband. Documed, Basel, 1990: 331-340

8. Walther H, Meyer FP. Klinische Pharmakologie antibakterieller Arzneimittel. Urban \& Schwarzenberg, München, 1987

9. Feldmann U, Schneider B, Klinkers H, Haeckel R. A multivariate approach for the biometric comparison of analytical methods in clinical chemistry. J Clin Chem Clm Biochem 1981; 19: 121-137

10. Passing H, Bablok W. A new biometrical procedure for testing the equality of measurements from two different analytical methods. Application of linear regression procedures for method comparison studies in clinical chemistry, Part I. J Clin Chem Clin Biochem 1983; 21: 709-720

11. Passing H, Bablok W. Comparison of several regression procedures for method comparison studies and determination of sample sizes. Application of linear regression procedures for method comparison studies in clinical chemistry, Part II. J Cln Chem Clin Biochem 1984; 22: 431-445

12. Sheiner LB, Beal SL. Some suggestions for measuring predictive performance. J Pharmacokinet Biopharm 1981; 9: 503-512 